\title{
Proteomics Analysis of the Cerebellum in Spontaneously Hypertensive Rats Treated with Twirling Reinforcing Manipulation, Twirling Reducing Manipulation or Electroacupuncture
}

\section{Xu-dong Zhang}

Beijing University of Chinese Medicine

\section{Xiao-min Hao}

Beijing University of Chinese Medicine College of Acupuncture and Massage Jing-rong Liang

Beijing University of Chinese Medicine College of Acupuncture and Massage Jiao-juan Wu

Beijing University of Chinese Medicine College of Acupuncture and Massage

\section{Yan-jun Xue}

Beijing University of Chinese Medicine College of Acupuncture and Massage

\section{Xiao-li Wu}

Beijing University of Chinese Medicine College of Acupuncture and Massage

\section{Peng-cheng Wei}

Acupuncture and Massage College Inc

\section{Yu Wang}

Beijing University of Chinese Medicine First Clinical Medical College

\section{Min Xiao}

Beijing University of Chinese Medicine First Clinical Medical College

\section{Ji-ping Zhao}

Beijing University of Chinese Medicine First Clinical Medical College

Qing-guo Liu (D liuqingguo999@sina.vip.com )

Beijing University of Chinese Medicine First Clinical Medical College

\section{Research}

Keywords: acupuncture, spontaneously hypertensive rat, proteomics, twirling reinforcing and reducing manipulation

Posted Date: November 5th, 2020 
DOl: https://doi.org/10.21203/rs.3.rs-101204/v1

License: (c) (1) This work is licensed under a Creative Commons Attribution 4.0 International License. Read Full License 


\section{Abstract}

Purpose: In this experiment, spontaneously hypertensive rats (SHR) were used to further explore the antihypertensive effect of acupuncture twirling reinforcing and reducing manipulation and the central effect mechanism of regulating blood pressure.

Methods: 32 male SHR rats were randomly divided into 4 groups: twirling reinforcing manipulation group $(R F)$, twirling reducing manipulation group (RD), electroacupuncture group $(E A)(n=8)$ and model group $(M)(n=8)$ and 8 WKY rats were used as blank control group (WKY). Group RD, RF and EA SHR took bilateral Taichong(LR3) point for acupuncture twirling method or electroacupuncture treatment, while WKY and $\mathrm{M}$ group did not use any acupuncture manipulation, but only carried out the same grasping and fixation. The differential proteins in cerebellum were screened by proteomic technology, and the key proteins and pathways involved in the pathogenesis of hypertension were selected.

Results: RD, RF and EA lowered the blood pressures of the SHRs by varying degrees. The results of Parallel reaction monitoring(PRM) verification showed that among all the differential proteins, 13 differentially expressed proteins (DEPs) were selected for PRM verification. The KEGG annotations of differential proteins are involved in the regulation of lipolysis, autophagy, gap junction, phosphate inositol metabolism, splicing, lysosome, thermogenesis and RNA transport in adipocytes, etc.

Conclusion: The results of the current study demonstrated that RD, RF and EA manipulations at the LR3 point reduced blood pressure and that RD was the most effective of the techniques used. Different acupuncture techniques have different pathways for screening and activating cerebellar differential proteins.

\section{Background}

Essential hypertension (essential hypertension, $\mathrm{EH}$ ) is the most common cardiovascular and cerebrovascular disease, which is mainly characterized by increased systemic arterial pressure, which can be accompanied by headache, vertigo, irritability and damage to heart, brain, kidney and other important target organs, seriously affecting human physical and mental health and quality of life. Although the angles and methods of traditional Chinese and western medicine in explaining and treating hypertension are different, the main goals are to prevent and control hypertension and to maintain stable and lasting blood pressure[1].

Acupuncture treatment has been reported to have a therapeutic effect on various diseases, particularly hypertension [2,3]. Previous studies have demonstrated that reinforcing and reducing manipulation was able to decrease blood pressure via multiple biological processes, including upregulation of nitric oxide synthase and cyclic guanosine monophosphate in arterial tissues [4], the brain network [5] and cell signal transduction pathways [6]. Positron emission tomography functional imaging the brains of rats who had receiving twirling reinforcing and reducing manipulation indicated that these acupuncture manipulations had central effects on mechanisms such as cerebral glucose metabolism [7]. Cerebellum serves an 
important role in the development and maintenance of hypertension. Different acupuncture manipulations activate different brain areas and these areas produce neurotransmitters that regulate blood pressure [8]. These results indicated that the brain exhibited core response to acupuncture manipulations and that neural protein expression, including the expression of related neurotransmitters, was impacted.

The field of proteomics is extremely active, and it has made a lot of very important progress in the field of life science, and it has gradually become one of the most effective methods to find disease molecular markers and drug targets. What's more, proteomics technology is an important technology to study the related mechanism of hypertension $[9,10,11]$. Proteomics plays a very important role in the clinical diagnosis, screening and treatment of hypertension, and provides a new platform and method for the study of hypertension.

The current study hypothesized that twirling reinforcing manipulation and twirling reducing manipulation, electroacupuncture may activate the cerebellum to release neurotransmitters by influencing protein expression profile, thus regulating blood pressure and that different acupuncture manipulations have different protein expression patterns, leading to differences in the effects on blood pressure.

\section{Materials And Methods}

\section{Animals.}

All animal experimental procedures were conducted in accordance with the World Health Organization's International Guiding Principles for Biomedical Research Involving Animals [12] and were approved by the Animal Care and Use Committee of Beijing University of Chinese Medicine, Beijing, China (permit no.BUCM-4-2018061501-2058). All rats were housed with 4 individuals per clean cage, with free access to water and food on a $12 \mathrm{~h} \mathrm{light/dark} \mathrm{cycle} \mathrm{at} \mathrm{a} \mathrm{humidity} \mathrm{of} 50-60 \%$ and at a temperature of $18-22^{\circ} \mathrm{C}$ during the process. A total of 32 SHR and 8 WKY male rats (age, 9 weeks; weight, $215 \pm 20 \mathrm{~g}$ ) were obtained from Beijing Vital River Laboratory Animal Technology Co., Ltd. SHRs were randomly divided into the model (M), twirling reinforcing manipulation (RF), twirling reducing manipulation (RD) and electroacupuncture (EA) groups ( $n=8 /$ group). In order to establish the model male WKY rats with continuous systolic blood pressures of $150-175 \mathrm{mmHg}$ were mated with female WKY rats with systolic blood pressures of 130-140 mmHg by Beijing Vital River Laboratory Animal Technology Co., Ltd. Offspring with systolic blood pressures $>150 \mathrm{mmHg}$ were obtained. Rats with high blood pressure were selected for inbreeding and the stable inheritance of hypertension was obtained by selective inbreeding for 20 generations and the SHR model was established [13, 14]. WKY rats were used as the normotensive control group of SHR and the control strain of hypertensive rats, as previously described [15].

\section{Sample preparation.}

All acupuncture manipulations were performed following 1 week of adaptation. The blood pressure of the WKY rats was relatively stable. SHRs were easily irritated and hyperactive; however, they were relatively 
quiet in the morning. The systolic blood pressure in the caudal artery was measured by two experienced technicians at a controlled temperature of $\left(20 \pm 2{ }^{\circ} \mathrm{C}\right)$. Each rat was gently placed in a restrainer and its tail was fixed using the rat-tail fixing facility. The ventral portion of each rat was placed on a heat pad, while the blood pressure measurement cuffs were put in place. Once a batch of 2 rats was in place, calm rats were preheated at $36^{\circ} \mathrm{C}$ for $10 \mathrm{~min}$, after which their systolic blood pressure was measured with a non-invasive blood pressure instrument (BP-6, Chengdu Thai Union Biological Instrument Co., Ltd., Chengdu, China [http://www.tme.com.cn/]) and recorded by the pulse recording sensor hile the rats were quiet and conscious. Each rat was measured three times and the mean value of the three times was taken as the systolic pressure. The systolic blood pressure of the rats was measured between 8:00 and 12:00 a.m. every four days between day 1 (one day prior the acupuncture experiment) and day 15 . Acupuncture at Taichong (LR3) was performed between 2:00 and 4:00 p.m. every day for 2 weeks. The 8 WKY (group WKY) and 32 SHRs (group M) did not undergo acupuncture; however, they were handled and held for 20 min in the same restrainer. Acupuncture on the SHRs in group RF, RD and EA was performed according to previous published studies [16]. Briefly, acupuncture needles were inserted to LR3 loci with respective acupuncture manipulations. For RF, the manipulation was performed with the forward thumb exerting heavy force, while the backward thumb exerted light force; meanwhile, for RD, the manipulation was conducted with the forward thumb exerting light fore with the backward thumb exerting with heavy force. The frequency and angle used were the same as a previous study [17]. EA was conducted according to another previous study [18]. The rats were treated once per day for 14 days. The same acupuncturist performed all acupuncture and sham treatments.

Cerebellum were collected for proteomics analysis. All experimental rats were sacrificed following anesthesia with an intraperitoneal injection of $3 \%$ pentobarbital sodium $(30 \mathrm{mg} / \mathrm{kg})$. The anesthetic regimen resulted in adequate anesthesia (Pinna reflex and pedal reflex were tested.) in all rats within 10 min $[19,20]$. Rats were sacrificed by cervical dislocation [21]. The cerebellum was isolated from each rat brain using tweezers and transferred to liquid nitrogen for preservation.

\section{Protein extraction and digestion.}

Cerebellum were homogenized three times at speed 6 for $40 \mathrm{sec}$ in $8 \mathrm{M}$ urea supplemented with cOmplete ${ }^{\mathrm{TM}}$ protease inhibitor cocktail (Roche Diagnostics) using a FastPrep instrument (MP Biochemicals, Inc.; Thermo Fisher Scientific, Inc.). The supernatant containing total protein was collected following centrifugation at $13,000 \times \mathrm{g}$ for $30 \mathrm{~min}$ at $4^{\circ} \mathrm{C}$. Concentrations were determined using a BCA assay kit (Thermo Fisher Scientific, Inc.) according to the manufacturer's protocol. A total of $150 \mu \mathrm{g}$ of protein from each sample was reduced with $10 \mathrm{mM}$ Tris-(2-carboxyethyl) phosphine (Thermo Fisher Scientific, Inc.) at $37^{\circ} \mathrm{C}$ for $60 \mathrm{~min}$ and alkylated with $40 \mathrm{mM}$ iodoacetamide (cat. no. A3221; SigmaAldrich; Merck, KGaA) in the dark at room temperature for $30 \mathrm{~min}$. Protein samples were collected using acetone precipitation. A volume of $400 \mu \mathrm{l}$ acetone was added to each sample which was then stored at $-20^{\circ} \mathrm{C}$ for 2 hours. Proteins was collected by centrifugation at $16,000 \mathrm{xg}$ at $4^{\circ} \mathrm{C}$ for $20 \mathrm{~min}$. Then the protein samples were digested with trypsin (Promega Corporation) at $37^{\circ} \mathrm{C}$ overnight. The peptides were desalted on Sep-Pak tC18 cartridges (Waters Corporation), according to the manufacturer's protocol. 


\section{Label-free proteomics analysis.}

Tryptic peptides were analyzed on a Q-Exactive mass spectrometer (Thermo Fisher Scientific, Inc.) coupled to an Easy-nLC 1200 nanoflow liquid chromatography system (Thermo Fisher Scientific, Inc.). The dried peptides were redissolved with Solvent A [ $2 \%$ acetonitrile and $0.1 \%$ trifluoroacetic acid (TFA; cat. no. 28903; Thermo Fisher Scientific, Inc.) in water] and $2 \mu \mathrm{g}$ was loaded onto a C18 column $(1.9 \mu \mathrm{m} ; 75 \times$ $25 \mathrm{~cm}$; Thermo Fisher Scientific, Inc.) and separated with a gradient of $5-38 \%$ Solvent B $(80 \%$ acetonitrile and $0.1 \%$ formic acid at a flow rate of $300 \mathrm{nl} / \mathrm{min}$. The data-dependent acquisition mode was used for the mass spectrometry (MS) for $120 \mathrm{~min}$. Full mass scans $(350-1800 \mathrm{~m} / \mathrm{z})$ were acquired at a resolution of 70,000 and the top 20 most abundant precursor ions were selected for higher-energy collisional dissociation fragmentation at a resolution of 17,500. The dynamic exclusion was $18 \mathrm{sec}$.

Proteome Discoverer software (version 2.2; Thermo Fisher Scientific, Inc.) was used for protein identification against the Rattus norvegicus UniProt database (https://www.uniprot.org/uniprot/? query=reviewed:yes\%20taxonomy:10114,28-08-2018). Cysteine reduced with iodoacetamide was set as the static modification and the oxidation of methionine and acetylation of the protein N-terminus were set as the dynamic modifications. The maximum missed cleavage sites and the precursor mass tolerance were set to 2 and $10 \mathrm{ppm}$, respectively. The cutoff of the global false discovery rate was set to 0.01 at the peptide level.

\section{Parallel reaction monitoring (PRM)-MS validation.}

Protein abundance differences obtained in the label-free proteomics analysis were confirmed using PRM assays. Independent retention time peptides (Biognosys AG) were added to the samples, according to the manufacturer's protocol. The scheduled PRM assays were conducted on the Q-Exactive HF coupled EasynLC 1,200 nanoflow liquid chromatography system (Thermo Fisher Scientific, Inc.). SpectroDive 9 software (version SW-3002;Biognosys AC) was used to determine the target protein and perform data analysis using the default parameters.

\section{Statistical analysis.}

Mixed ANOVA and the Bonferroni's post-hoc test was performed to detect differences in blood pressure. Label-free and PRM data were analyzed using one-way ANOVA followed Tukey's post-hoc test. The Venn diagram was drawn using R version 3.6.3 (VennDiagram package). Gene ontology (GO) annotation was performed using Blast2GO® (www.blast2go.com) and GOATOOLS

(v0.6.10, https://github.com/tanghaibao/goatools/releases/tag/v0.6.10) was used to run the GO enrichment analysis. $P$ values were assessed using Fisher's exact test, and adjusted with BenjaminiHochberg method. Az-score algorithm was used to predict the function of the DEPs. A size of 35 focus molecules, both direct and indirect relationships with Fisher's exact test were applied to run the network analysis (score =-log ( $p$-value)). One-way ANOVA and Bonferroni's post-hoc test was performed for PRM data analysis. Graphs of PRM data were prepared using GraphPad Prism (version 6; GraphPad Software, Inc.). All statistical analysis was performed using SPSS( version 22; IBM Corp.) 


\section{Results}

\section{Multiple acupuncture manipulations lower blood pressure in SHRs.}

To study the antihypertensive effects of different acupuncture manipulations, RF, RD and EA was performed on SHRs. The systolic blood pressure of all experimental rats was measured regularly (Table I). The results demonstrated that there was no significant difference in the blood pressure measurements between the acupuncture-treated groups (RD, RF and $E A$ ) and group $M($ all, $P>0.05$ ) on day 0 (one day prior to acupuncture manipulation). However, the blood pressure measurements of the M, RD, RF and EA groups were significantly higher compared with group WKY at all time points (all $\mathrm{P}<0.05$ ). The blood pressure measurements of the RD, RF and EA groups decreased significantly compared with the M group on days 8,12 and 14 (all, $P<0.05$ ). All three acupuncture manipulation groups exhibited attenuated blood pressures following 14 days of treatment; however, the blood pressure measurements of the RD, RF and EA groups were reduced by varying degrees. The blood pressure measurements of the RD group were lower compared with the RF and EA groups on day 14; however, the differences were not significant (both, $P>0.05)$. These results indicated that the acupuncture manipulations had a positive effect in lowering the blood pressure measurements of the SHR groups, with RD possibly being the most effective treatment. The blood pressure measurements of the RD group declined over time and there was a significant difference at day 12 vs. day 4 and day 14 vs. day 4 (both, $P<0.05$ ). There was a slight decrease in the blood pressure measurements in the RD and EA groups; however, $\mathrm{P}>0.05$ in all cases (all the others days vs. day 0 ). These data indicated that RD, RF and EA lowered the blood pressures of the SHRs by varying degrees. Furthermore, the data revealed an instant and long-term protective effect of RD and an instant effect of RF and EA in SHRs.

Table I Comparison of systolic blood pressure ( $x \pm S, \mathrm{mmHg})$

\begin{tabular}{|c|c|c|c|c|c|c|}
\hline Group & $\mathbf{N}$ & Day0 & Day4 & Day8 & Day12 & Day14 \\
\hline WKY & 8 & $\begin{array}{l}109.93 \pm \\
1.82\end{array}$ & $\begin{array}{l}111.07 \pm \\
2.43\end{array}$ & $111.57 \pm 2.41$ & $111.86 \pm 2.74$ & $111.50 \pm 3.01$ \\
\hline$M$ & 8 & $\begin{array}{l}166.07 \pm \\
1.33 a\end{array}$ & $\begin{array}{l}165.71 \pm \\
2.67 a\end{array}$ & $169.14 \pm 2.28 a$ & $171.43 \pm 2.06 a$ & $173.79 \pm 2.15 a$ \\
\hline $\mathrm{RF}$ & 8 & $\begin{array}{l}166.14 \pm \\
2.14 a\end{array}$ & $\begin{array}{l}164.29 \pm \\
1.64 a\end{array}$ & $\begin{array}{l}164.07 \pm \\
2.70 a b\end{array}$ & $\begin{array}{l}163.43 \pm \\
3.01 \mathrm{ab}\end{array}$ & $\begin{array}{l}163.07 \pm \\
2.84 a b\end{array}$ \\
\hline $\mathrm{RD}$ & 8 & $\begin{array}{l}166.21 \pm \\
1.71 a\end{array}$ & $\begin{array}{l}163.93 \pm \\
2.76 a\end{array}$ & $\begin{array}{l}162.07 \pm \\
3.08 \mathrm{abc}\end{array}$ & $\begin{array}{l}160.79 \pm \\
2.33 a b c\end{array}$ & $\begin{array}{l}158.71 \pm \\
2.84 a b c\end{array}$ \\
\hline EA & 8 & $\begin{array}{l}166.00 \pm \\
1.92 a\end{array}$ & $\begin{array}{l}164.07 \pm \\
3.58 a\end{array}$ & $\begin{array}{l}162.71 \pm \\
2.52 \mathrm{ab}\end{array}$ & $\begin{array}{l}160.86 \pm \\
2.82 \mathrm{abc}\end{array}$ & $\begin{array}{l}159.93 \pm \\
3.32 \mathrm{abc}\end{array}$ \\
\hline
\end{tabular}




\section{Analyses of the proteomics data.}

To investigate the effect of acupuncture treatment on the central nervous system, proteomics analysis on the cerebellum of the SHRs was performed. Cerebellum from all rats were subjected to proteomics analysis through a label-free technique to obtain profiling data. The results identified 4,378 protein groups (Fig. 1A). Following this, four comparisons (M/WKY, RF/M, EA/M and RD/M) were performed to identify altered proteins. A total of 397(M/WKY), 96(RF/M), 133 (EA/M) and 216 (RD/M) proteins were identified as DEPs $(P<0.05$; fold change $\geq 1.2)$. Further comparisons were performed to investigate the differences and overlaps in the DEPs induced by the RD, RF and EA manipulations via Venn analysis (Fig. 1B), indicating the presence of numerous rescue effect-related proteins. GO enrichment analysis of DEPs in the groups RF, EA RD and WKY were compared with the M group. Following this, volcano and GO enrichment analyses of these proteins was conducted. (Fig. 1C, 1D,1E)Extracellular exosome and endoplasmic reticulum membrane were among the top 5 most significant $(P<0.05)$ terms in all of the GO enrichment analyses, indicating that hypertension and acupuncture manipulations affected the expression of secreted proteins in the cerebellum of the SHRs. In summary, these results may elucidate the effect of acupuncture manipulation on cerebellum in the SHRs.

\section{Analyses of KEGG pathway enrichment and protein interoperability network}

To identify biological processes most relevant to biological phenomena, we did the analyses of KEGG pathway enrichment and protein interoperability network. There were $41 \mathrm{KEGG}$ pathways significantly enriched $(P<0.05)$ in $M$ compared with WKY group(Fig. $2 A)$ : CAMP signaling pathway, HIF-1 signaling pathway, mTOR signaling pathway, Ras signaling pathway, Huntington's disease,Alzheimer's diseaseThuman immunodeficiency virus type 1 infection, nonalcoholic fatty liver disease (NAFLD), Parkinson's disease, herpesvirus infection, Kaposi's sarcoma-associated herpesvirus infection, alcoholism, viral carcinogenesis, chemical carcinogenesis, human t cell leukemia virus 1 infection, tuberculosis, anti-folate resistance, hypertrophic cardiomyopathy (HCM), insulin resistance, oxidative phosphorylation, retinol metabolism, pyrimidine metabolism, folate biosynthesis, purine metabolism, other polysaccharide degradation, nicotinic acid and nicotinamide metabolism, steroid biosynthesis, Dglutamine and D-glutamic acid metabolism, sphingolipid metabolism, thermogenesis, glucagon signaling pathway, hematopoietic cell lineage, estrogen signaling pathway, thyroid hormone signaling pathway, cholesterol metabolism, gastric acid secretion, ribosome, spliceosome, endoplasmic reticulum protein processing, cell iron death, oocyte maturation division. There were respectively $12(R F / M), 11(R D / M)$, 15(EA/M) pathways with significant differences in protein enrichment(Fig. 2B,2C,2D). The overall trend of PRM verification results and Lable free quantitative results is consistent in this experiment. The KEGG annotation of these differential proteins in adipocyte lipolysis regulation, autophagy, gap junction, phosphoinositide metabolism, spliceosome, lysosome, thermogenesis and RNA transport. A total of 397 significant differential proteins was more than 1.2 or less than 0.83 times in Group WKY compared with M group. Furthermore, a total of 96, 216, 133 differentially expressed proteins with up and down 
regulation of more than 1.2 fold or less than 0.83 were screened in Group RF, RD, EA compared with $M$ group Of all the differential proteins.(Fig. 3A,3B,3C,3D), 13 target proteins were selected for PRM verification. It can be found from the table that the PRM verification results are consistent with the overall trend of Lable free quantitative results, including Acsl6(P33124), Kifap3(A0A0G2K0P1), Atat1(Q6MG11), Pkg(A9LNM8), Rab23(D3ZRM5), Zfyve1(D4A3T4), Nup205(D4A7R3), Apoa2(P04638), Nrbp1(Q3SWT7), Gga1(Q5FVF3), Eif3(Q5RK09), Clint1(Q6DGF2) and Pi4k2ag(Q99M64).(Table区)

Table冈 Description of 13 target proteins

\begin{tabular}{|lll|}
\hline $\begin{array}{l}\text { Protein } \\
\text { Number }\end{array}$ & $\begin{array}{l}\text { Gene } \\
\text { Name }\end{array}$ & Protein Description \\
\hline P33124 & Acs16 & long chain fatty acid A coenzyme 6 \\
\hline A0A0G2K0P1 & Kifap3 & Kallikrein 3 \\
\hline Q6MG11 & Atat1 & a-tubulin N-acetyltransferase1 \\
\hline A9LNM8 & Pkg & cGMP-dependent protein kinases \\
\hline D3ZRM5 & Rab23 & RAB 23,RAS oncogene family members \\
\hline D4A3T4 & Zfyve1 & Zinc-containing finger FYVE 1 \\
\hline D4A7R3 & Nup205 & Nuclear pore size 205 \\
\hline P04638 & Apoa2 & A-Il of apolipoprotein \\
\hline Q3SWT7 & Nrbp1 & Nuclear receptor-binding protein \\
\hline Q5FVF3 & Gga1 & $\begin{array}{l}\text { Golgi-associated ,y aptamer ear-containing agent, ARF binding } \\
\text { protein 1 }\end{array}$ \\
\hline Q5RK09 & Eif3g & Eukaryotic translation initiation factor 3 subunit G \\
\hline Q6DGF2 & Clint1 & Cage protein interaction factor \\
\hline Q99M64 & Pi4k2a & phosphatidylinositol 4-kinase 2-a \\
\hline
\end{tabular}

\section{Discussion}

Numerous experimental and case studies have indicated that acupuncture manipulation lowered the blood pressure measurements of SHRs and patients with hypertension [22] and LR3 was the most frequently used location in clinical trials $[23,24]$. In previous studies, RD, RF and EA at the LR3 location lowered the blood pressure measurements of SHRs and affected vascular smooth muscle through a central mechanism involving activated key brain regions $[25,26]$.

Acupuncture twisting and reinforcing diarrhea and electroacupuncture can significantly reduce SHR blood pressure and reduce brain injury. The antihypertensive effect of twisting and diarrhea method is the most obvious and the amplitude of antihypertensive is the most significant. Different acupuncture techniques 
have different pathways for the screening and activation of cerebellar differential proteins. The pathogenesis of SHR rats may be closely related to Ras signaling pathway, oxidative phosphorylation pathway, mTOR signaling pathway, cAMP signaling pathway and insulin resistance pathway. Antihypertensive mechanism of acupuncture may be closely related to vascular smooth muscle contraction, VEGF signaling pathway, oxidative phosphorylation and gap junction pathway. In the present study, the antihypertensive effects of three acupuncture manipulations (RD, RF and EA) on LR3 were compared to further reveal the central mechanism underlying the antihypertensive effect of acupuncture treatments, proteomics analysis was performed to examine the protein expression in the cerebellum, followed by a PRM experiment for validation. The results demonstrated that different acupuncture treatments lowered blood pressure to varying degrees and multiple dysregulated proteins and potential networks associated with acupuncture stimuli were identified.

The previous research found that acupuncture can achieve hypotensive effect by regulating neurotransmitters in the heart, brain and kidney. Cerebellum plays an important role in regulating cardiovascular function in addition to regulating motor activity, muscle tension and maintaining balance. Many early studies found[27, 28], electrical stimulation of the cerebellar parietal nuclear energy caused a significant increase in arterial pressure, and the cerebellum was associated with cardiovascular activity Meridian, play an important role in cardiovascular function; acupuncture LR3 activated brain area mainly concentrated in cerebellum-posterior lobe, cingulate gyrus, dorsal thalamus and frontal lobe combined cortex and other ; acupuncture can regulate the cerebellum NE囚5-HTDNO achieve hypotension effect [29].The main function of the cerebellum is to participate in body balance, coordinate random movement and regulate muscle tone. Recent studies have found that the cerebellum not only plays a role in cognitive, emotional and other advanced brain functions, but also plays a very important role in regulating blood pressure[30]. In the pressor response of the fastigial nucleus, it was found that stimulating the oral part of the cerebellar fastigial nucleus could cause obvious cardiovascular response, indicating that the cerebellum could regulate blood pressure in some ways.

In conclusion, the results of the current study demonstrated that RD, RF and EA manipulations at the LR3 point reduced blood pressure measurements and that RD was the most effective of the techniques used. The cerebellum protein expression profiles of the SHRs were altered following acupuncture therapy. Furthermore, multiple networks and proteins were identified and they may represent potential antihypertensive drug targets.

\section{Conclusion}

In this study, the antihypertensive effects of different acupuncture methods were different, and the screening and activation pathways of cerebellar proteins were also different. This helps to choose the best acupuncture method for the treatment of hypertension. On the other hand, different acupuncture methods have different expressions of cerebellar protein. This clarifies the central mechanism of hypertension to some certain extent, and further arouses people's attention to the cerebellar mechanism 
of hypertension. The above conclusions can confirm the different effects of different acupuncture therapies on hypertension and the important role of the cerebellum in the pathogenesis of hypertension.

\section{Abbreviations}

$\mathrm{EH}=$ essential hypertension, $\mathrm{SHR}=$ spontaneously hypertensive rats, $\mathrm{WKY}=$ Wistar-Kyoto Rat, RF = twirling reinforcing manipulation group, $\mathrm{RD}=$ twirling reducing manipulation group, $\mathrm{EA}=$ electroacupuncture group, $\mathrm{PRM}=$ Parallel reaction monitoring, $\mathrm{GO}=$ Gene ontology, $\mathrm{DEPs}=$ differentially expressed proteins, KEGG = Kyoto Encyclopedia of Genes and Genomes, NAFLD = nonalcoholic fatty liver disease, $\mathrm{HCM}$ = hypertrophic cardiomyopathy.

\section{Declarations}

\section{Acknowledgements}

Not applicable.

\section{Funding}

The current study was supported by Chinese Nature Science Foundation Grants (grant no. 81774413).

\section{Availability of data and materials}

All the thermo raw files of proteomics analysis can be obtained from iProx database (https://www.iprox.org/page/HMV006.html, accession number IPX0002465001).

\section{Author's contributions}

Xu-dong Zhang and Xiao-min Hao wrote the paper and was responsible for the design and running of the main experiments. Qing-guo Liu was responsible for the guidance in the experiment. Jing-rong Liang and Jiao-juan Wu measured the blood pressure of rats. Yan-jun Xue, Xiao-li Wu and Peng-cheng Wei performed acupuncture manipulations. Yu Wang, Ji-ping Zhao and Min Xiao analyzed the data. All authors read and approved thefinal version of the paper.

\section{Ethics approval and consent to participate}

All experimental procedures were approved by the Animal Care and Use Committee Of Beijing University of Chinese Medicine, Beijing, China (permit no. BUCM-4-2018061501-2058).

\section{Patient consent for publication}

Not applicable.

\section{Competing interests}


The authors declare that they have no competing interests.

This study was supported by the National Natural Science Foundation of China (No. 81774413).The funders had no participation in study design, data collection and analysis, decision to publish, or preparation of the manuscript.

The authors have no conflicts of interests to disclose.

\section{References}

1. John M. Flack, Bemi Adekola. Blood pressure and the new ACC/AHA hypertension guidelines. 2020, 30(3):160-164.

2. Zhu, D.C., et al., [Thermosensitive Moxibustion Induces A Better Therapeutic Effect in the Treatment of Facial Paralysis Patients]. Zhen Ci Yan Jiu, 2018. 43(10):666-9.

3. Xu,L.,et al., [Treatment of knee osteoarthritis with acupuncture and moxibustion: a randomized controlled trial]. Zhongguo Zhen Jiu, 2013. 33(10):871-6.

4. Liu, W., et al., [Effect of twirling-reinforcing-reducing needling manipulations on contents of serum acetylcholine and arterial NOS and cGMP in stress-induced hypertension rats]. Zhen Ci Yan Jiu, 2015. 40(2): 136-40.

5. Chen, H., et al., Hypothalamus-related resting brain network underlying short-term acupuncture treatment in primary hypertension. Evid Based Complement Alternat Med, 2013. 2013: 808971.

6. Cheng, L., et al., What do we understand from clinical and mechanistic studies on acupuncture treatment for hypertension? Chin Med, 2015. 10: 36.

7. Li, J., et al., Effect of Acupuncture at LR3 on Cerebral Glucose Metabolism in a Rat Model of Hypertension: A (18)F-FDG-PET Study. Evid Based Complement Alternat Med, 2018. 2018: 5712857.

8. Lai, X., et al., Proteomic response to acupuncture treatment in spontaneously hypertensive rats. PLoS One, 2012. 7(9): e44216.

9. Wang he, et al." effect of acupuncture twisting and reinforcing diarrhea on cerebellar NEख5-HT and NO in spontaneously hypertensive rats. Universal Chinese Medicine 2018;11(03):353-357.

10. Thongboonkerd V. Genomics, proteomics and integrative "omics" in hypertension research. Curr Opin Nephrol Hypertens. 2005;14(2):133-139.

11. Carty DM, Schiffer E, Delles C. Proteomics in hypertension. J Hum Hypertens. 2013;27(4):211-216.

12. Tyther $R$, McDonagh $B$, Sheehan $D$. Proteomics in investigation of protein nitration in kidney disease: technical challenges and perspectives from the spontaneously hypertensive rat. Mass Spectrom Rev. 2011;30(1):121-141.

13. Jones-Bolin, S., Guidelines for the Care and Use of Laboratory Animals in Biomedical Research. Current Protocols in Pharmacology, 2012. 59(1): p.A.4B.1-A.4B.9.

14. Okamoto, K. and K. Aoki, Development of a strain of spontaneously hypertensive rats. Jpn Circ J, 1963. 27: 282-93. 
15. Conn, P.M., Animal models for the study of human disease. Second edition. ed. 493xix, 1177 pages.

16. Okamoto, K., et al., Further observations of the development of a strain of spontaneously hypertensive rats. Jpn Circ J, 1966. 30(6): 703-16.

17. Guo, Q., et al., Twirling reinforcing-reducing manipulation-central mechanism underlying antihypertensive effect on spontaneous hypertension in rats. J Tradit Chin Med, 2018. 38(3): 391398.

18. Guo, Y., et al., Effect of electro-acupuncture on gene expression in heart of rats with stress-induced pre-hypertension based on gene chip technology. J Tradit Chin Med, 2015. 35(3): 285-94.

19. Yi-Ming, W., et al., Asynchronism of the recovery of baroreflex sensitivity, blood pressure, and consciousness from anesthesia in rats. J Cardiovasc Pharmacol, 2004. 43(1): 1-7.

20. Redfors, B., Y. Shao, and E. Omerovic, Influence of anesthetic agent, depth of anesthesia and body temperature on cardiovascular functional parameters in the rat. Lab Anim, 2014. 48(1): 6-14.

21. Xiang, B., et al., miR-183 inhibits microglia activation and expression of inflammatory factors in rats with cerebral ischemia reperfusion via NF-kappaB signaling pathway. Exp Ther Med, 2019. 18(4): p. 2540-2546.

22. Wang, J., X. Xiong, and W. Liu, Acupuncture for essential hypertension. Int J Cardiol, 2013. 169(5): $317-26$.

23. Zhao, X.F., et al., Is Acupuncture Effective for Hypertension? A Systematic Review and Meta-Analysis. PLoS One, 2015. 10(7): e0127019.

24. Wang, Y., et al., Cerebral Targeting of Acupuncture at Combined Acupoints in Treating Essential Hypertension: An Rs-fMRI Study and Curative Effect Evidence. Evid Based Complement Alternat Med, 2016. 2016: p. 5392954.

25. Qiulei, G., et al., Twirling reinforcing-reducing manipulation on upoints in Treating Essential Hypertension: An Rs-fMRn spontaneous hypertension in rats. Journal of Traditional Chinese Medicine, 2018. 38(3).

26. Nisimaru N . Cardiovascular Modules in the Cerebellum[J]. Japanese Journal of Physiology, 2004, 54(5):431-448.

27. Rocha I, Gonçalves V, Bettencourt MJ,et al. Effect of stimulation of sublobule IX-b of the cerebellar vermis on cardiac function[J]. physiological research, 2008, 57(5):701.

28. Wang he, et al. Effect of acupuncture twirling and reinforcing diarrhea on cerebellar NE囚5-HT and NO in spontaneously hypertensive rats Universal Chinese Medicine 11(03):353-357.

29. Joaquim AF. Severe Cerebellar Degeneration and Chiari I Malformation-Speculative pathophysiology based on a systematic review. Rev Assoc Med Bras (1992). 2020;66(3):375-379.

\section{Figures}




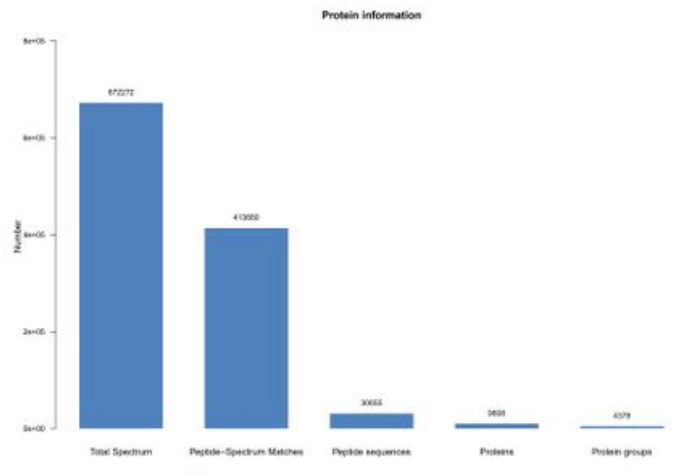

$1 \mathrm{~A}$
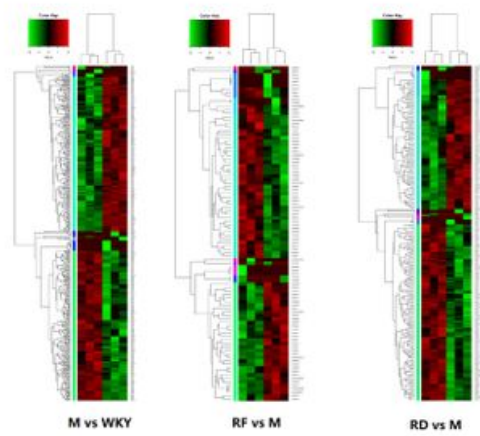

10

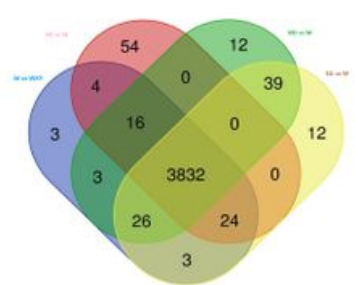

18

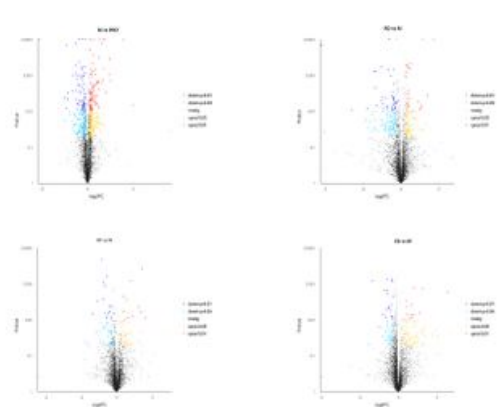

$1 C$

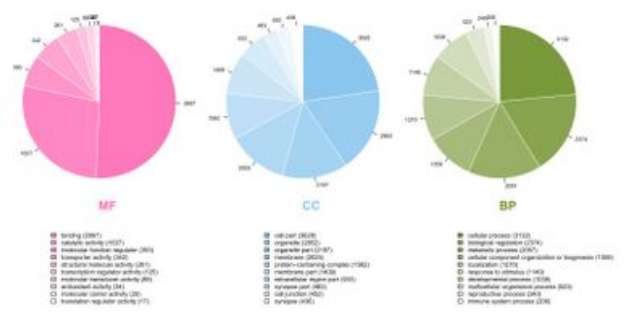

$1 E$

\section{Figure 1}

1A The results of protein information 1B Venn analysis 1C Volcano of DEPs 1D Heatmap of differentially expressed proteins in the four comparison groups (M/WKY, RF/M, RD/M, EA/M) 1E GO enrichment analysis of DEPs 


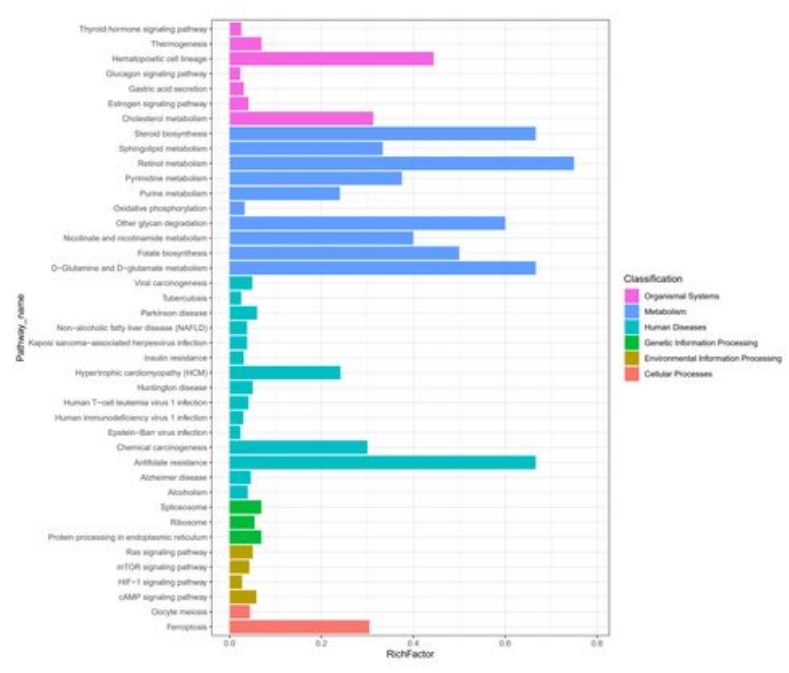

$2 \mathrm{~A}$

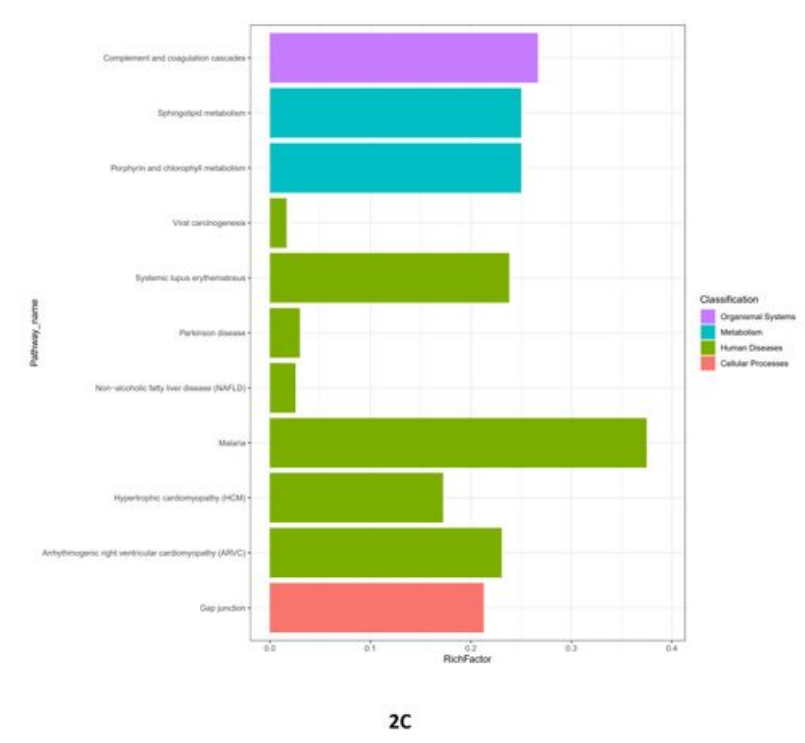

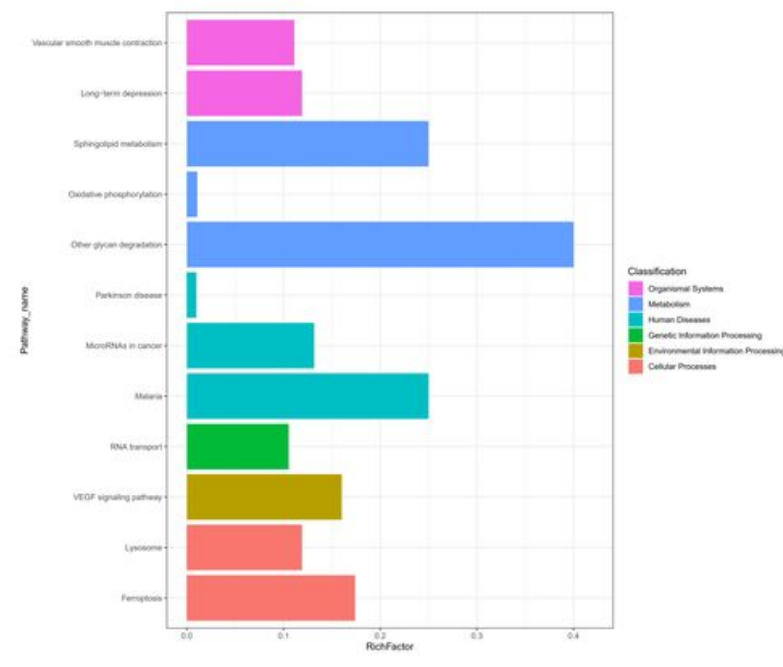

2B

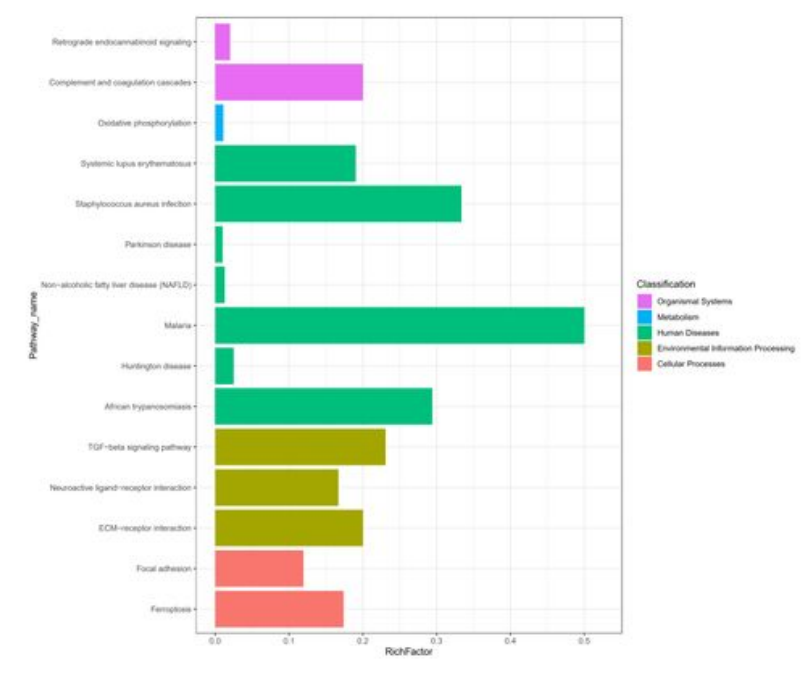

2D

Figure 2

2A KEGG pathway enrichment in M vs WKY 2B KEGG pathway enrichment in RF vs M 2C KEGG pathway enrichment in RD vs M 2D KEGG pathway enrichment in EA vs $M$ 


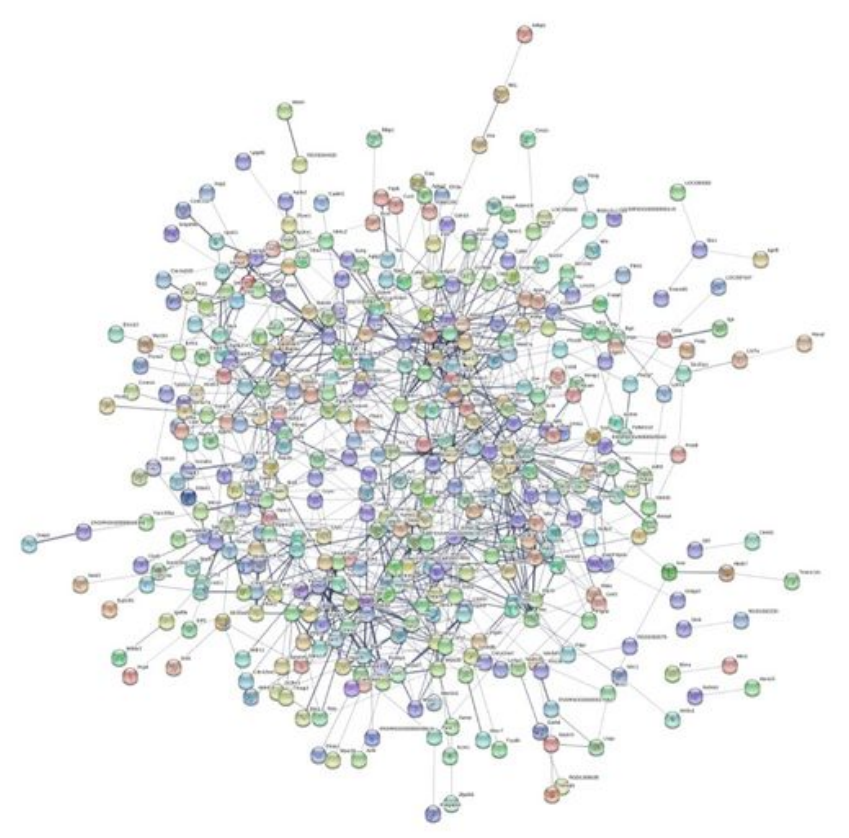

$3 a$

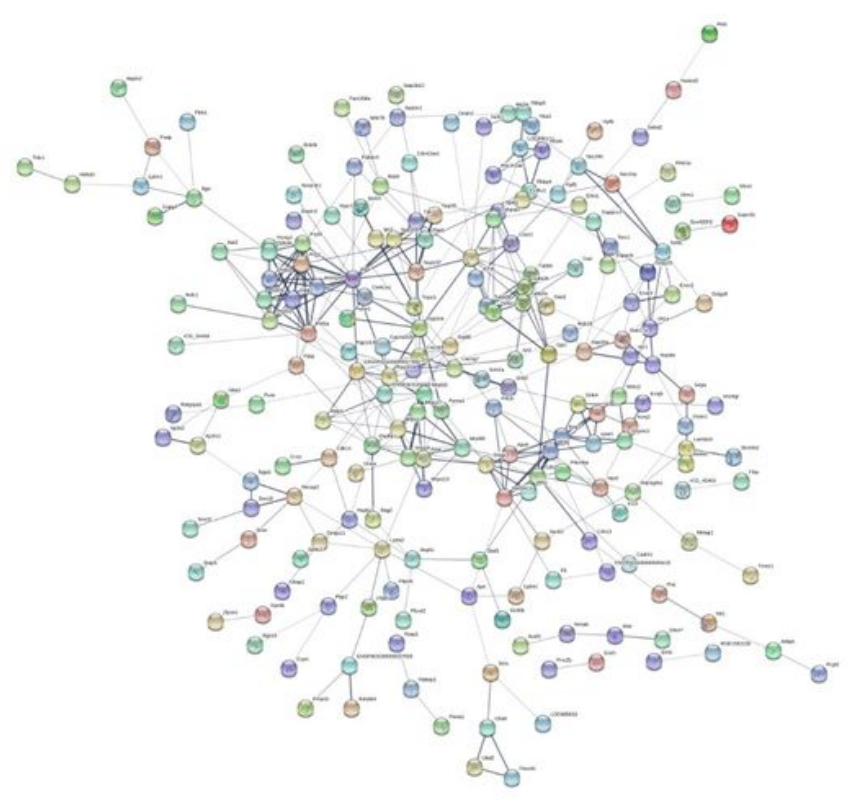

$3 c$

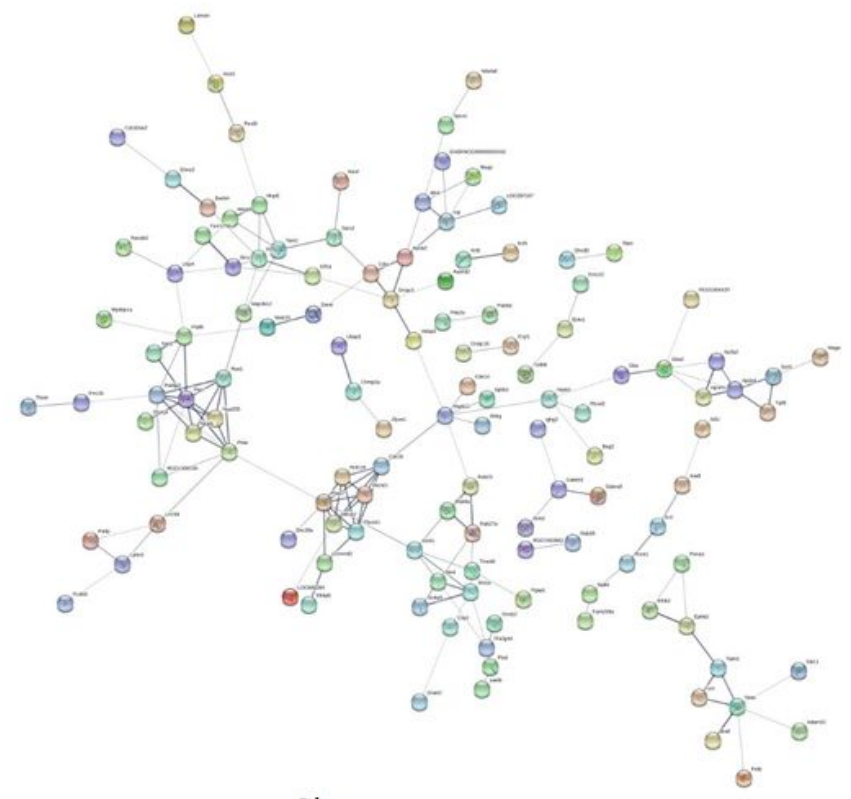

$3 b$

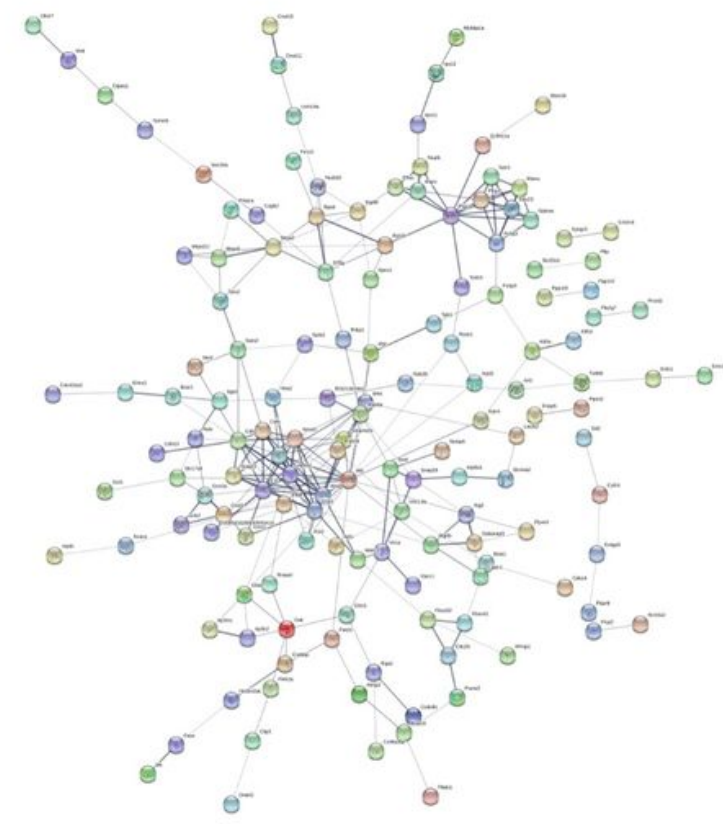

$3 d$

\section{Figure 3}

3A protein interoperability network in M vs WKY 3B protein interoperability network in RF vs M 3C protein interoperability network in RD vs $M 3 D$ protein interoperability network in EA vs $M$ 